\title{
BMJ Open Risk factors and complications of lower limb cellulitis in Africa: a systematic review
}

\author{
Frank-Leonel Tianyi, ${ }^{1}$ Clarence Mvalo Mbanga, ${ }^{2}$ Celestin Danwang, ${ }^{3}$ \\ Valirie Ndip Agbor ${ }^{4}$
}

To cite: Tianyi F-L, Mbanga CM, Danwang C, et al. Risk factors and complications of lower limb cellulitis in Africa: a systematic review. BMJ Open 2018;8:e21175. doi:10.1136/ bmjopen-2017-021175

- Prepublication history and additional material for this paper are available online. To view these files, please visit the journal online (http://dx.doi. org/10.1136/bmjopen-2017021175).

F-LT and CMM contributed equally.

Received 15 December 2017

Revised 7 April 2018

Accepted 8 June 2018

A Check for updates

C Author(s) (or their employer(s)) 2018. Re-use permitted under CC BY-NC. No commercial re-use. See rights and permissions. Published by BMJ.

${ }^{1}$ Department of General Medicine, Mayo Darley Subdivisional Hospital, Banyo, Cameroon

${ }^{2}$ Department of General Medicine, Mankon Subdivisional Hospital, Bamenda, Cameroon

${ }^{3}$ Department of Surgery and Specialties, Faculty of Medicine and Biomedical Sciences, University of Yaoundé I, Yaoundé, Cameroon

${ }^{4}$ Department of General Medicine, Ibal Sub-divisional Hospital, Oku, Cameroon

Correspondence to Dr Valirie Ndip Agbor; nvagbor@gmail.com

\section{ABSTRACT}

Objective To summarise available data on the risk factors, complications and the factors associated with complications of lower limb cellulitis in Africa.

Methods We did electronic searches on PubMed, EMBASE, Scopus and African Journals Online from 1 January 1986 to 30 October 2017, extracted and summarised data on the risk factors, complications and the factors associated with the complications of lower limb cellulitis from eligible literature.

Results A total of seven studies were retained for final review after the search and screening processes. Local risk factors of cellulitis reported were: disruption of the skin barrier, neglected wounds, toe-web intertrigo, leg ulcers, use of depigmentation drugs and leg oedema. Obesity was the only reported general risk factor of cellulitis. Five studies reported on the complications of cellulitis which included: abscess formation, necrotising fasciitis, bullae, haemorrhagic lesions, necrosis, phlebitis and amputations. Nicotine addiction, chronic use of non-steroidal anti-inflammatory drugs, delay in the initiation of antibiotic treatment and elevated erythrocyte sedimentation rate were risk factors of complications of lower limb cellulitis identified from three studies. Conclusion This review highlights the important role of local risk factors in the pathogenesis of lower limb cellulitis in Africa. The association between voluntary skin depigmentation and lower limb cellulitis should alert public health authorities and the general population to the health risks associated with this practice. The identification and improved management of the risk factors of lower limb cellulitis and its complications could go a long way in decreasing the morbidity and health costs incurred by lower limb cellulitis in Africa.

\section{INTRODUCTION}

Cellulitis is a skin and soft-tissue infection caused most often by Streptococci. ${ }^{12}$ It is characterised by inflammatory signs such as pain, swelling, redness and warmness of the affected area and/or systemic signs like malaise, fever, nausea or vomiting. ${ }^{134}$ Cellulitis affects the lower limbs in $88 \%$ of cases. ${ }^{5}$

Lower limb cellulitis is rarely fatal and usually responds well to proper antibiotherapy. ${ }^{6}$ Nevertheless, a delay in its diagnosis

\section{Strengths and limitations of this study}

- All of the studies included in this review were hospital based, limiting inference of our findings to the general population. This limited the cases of cellulitis to those deemed severe enough by patients to require health intervention.

- In addition, the very small number of included studies and the exclusion of four potentially eligible studies as their full texts could not be retrieved is likely to skew the findings presented in this review.

- Over $30 \%$ of cases of cellulitis have an alternative diagnosis. This is a potential limitation to our study, especially from retrospective studies.

- This is the first systematic review summarising the risk factors, complications and the predictors of complications of lower limb cellulitis in Africa.

- The inclusion of recent studies provides a contemporaneous picture of the risk factors and complications of cellulitis in Africa.

and appropriate treatment could lead to life-threatening and debilitating complications such as necrotising hypodermitis, necrotising fasciitis, abscess formation, septic shock and even death in extreme cases. ${ }^{7-9}$ Cellulitis is associated with a prolonged hospital stay, considerable increase in the mortality rate and years of healthy life lost in Africa. ${ }^{1011}$ All this cumulate to a reduction in income, aggravation of poverty levels and reduction in economic productivity, hindering socioeconomic development and achievement of the sustainable development goals 1,2 and $3{ }^{12}$

The risk factors of lower limb cellulitis are multiple, and can be grouped into general and local risk factors. General risk factors include obesity, diabetes, history of cellulitis, immunosuppression, chronic alcoholism and chronic use of non-steroidal anti-inflammatory drugs (NSAIDs), while disruption of the skin barrier, neglected wounds, toe-web intertrigo, leg ulcers, use of depigmentation drugs and leg oedema, were the most commonly reported local risk factors. ${ }^{13-16}$ 
Proper understanding of the risk factors and factors associated with the complications of lower limb cellulitis in Africa will help guide policy-makers and healthcare professionals in implementing preventive strategies, and consequently curb both the financial and health burden associated with the disease. Hitherto, no review has been conducted on lower limb cellulitis in Africa. This review sought to summarise existing data on the risk factors, complications and the factors associated with the complications of lower limb cellulitis in Africa.

\section{Objective}

To summarise available data on the risk factors, complications and the factors associated with complications of lower limb cellulitis in Africa.

\section{Review questions}

1. What are the risk factors of lower limb cellulitis in Africa?

2. What are the complications of lower limb cellulitis in Africa?

3. What are the factors associated with complications of lower limb cellulitis in Africa?

\section{METHODS}

The Preferred Reporting Items for Systematic Reviews and Meta-analyses guidelines was used to report this review. ${ }^{17}$

\section{Criteria for considering studies for the review}

\section{Inclusion criteria}

All studies done on humans possessing the following criteria were considered for the review:

1. Observational studies (case control, cross-sectional, case series with more than 30 participants and cohort studies) reporting on the risk factors, complications and factors associated with complications of lower limb cellulitis among African patients.
2. Participants aged 15 years and older and residing in Africa.

3. Published literature from 1 January 1986 to 31 October 2017 were considered for this review, with no language restrictions.

\section{Exclusion criteria}

We excluded:

1. Commentaries, letters to the editors and editorials.

2. Studies conducted on patients of African origin but residing outside the continent.

3. Studies reporting on cellulitis in other body areas like the face, neck and eye.

4. For duplicate studies, only the most recent and/or comprehensive version with the largest sample size was used.

\section{The literature search}

Medical subject headings and text words such as 'cellulitis' or 'erysipelas' or 'dermohypodermitis' were used to search the following electronic databases for relevant articles: PubMed, EMBASE, Scopus and African Journals Online. The individual nomenclature of all the 54 African countries was equally included in the search to increase its specificity and sensitivity. ${ }^{18}$ The search period spanned from 1 January 1986 to 30 October 2017, with no language restrictions. The main search strategy is depicted in table 1 .

The titles and abstracts of potential articles were reviewed and their full texts retrieved through PubMed, Google scholar, Scopus or the journal's website. Full texts of articles which could not be retrieved online were requested from the corresponding author via email. Studies were automatically excluded from the review if no reply was obtained from the corresponding author. The reference lists of eligible articles were then scrutinised to retrieve eligible articles missed during the search, and their full texts recovered as detailed above.

\section{Table 1 Main search strategy for PubMed}

\begin{tabular}{ll}
\hline SN & Search items \\
\hline 1. & (Cellulit* OR Erysipel $^{*}$ OR dermohypodermit ${ }^{\star}$ OR cellulitis (MeSH))
\end{tabular}

2. (Africa* OR Algeria OR Angola OR Benin OR Botswana OR "Burkina Faso" OR Burundi OR Cameroon OR "Canary Islands" OR "Cape Verde" OR "Central African Republic" OR Chad OR Comoros OR Congo OR "Democratic Republic of Congo" OR Djibouti OR Egypt OR "Equatorial Guinea" OR Eritrea OR Ethiopia OR Gabon OR Gambia OR Ghana OR Guinea OR "Guinea Bissau" OR "Ivory Coast" OR "Cote d'Ivoire" OR Jamahiriya OR Kenya OR Lesotho OR Liberia OR Libya OR Madagascar OR Malawi OR Mali OR Mauritania OR Mauritius OR Mayotte OR Morocco OR Mozambique OR Namibia OR Niger OR Nigeria OR Principe OR Reunion OR Rwanda OR "Sao Tome" OR Senegal OR Seychelles OR "Sierra Leone" OR Somalia OR "South Africa" OR "South Sudan" OR "St Helena" OR Sudan OR Swaziland OR Tanzania OR Togo OR Tunisia OR Uganda OR "Western Sahara" OR Zaire OR Zambia OR Zimbabwe OR "Central Africa" OR "Central African" OR "West Africa" OR "West African" OR "Western) Africa" OR "Western African" OR "East Africa" OR "East African" OR "Eastern Africa" OR "Eastern African" OR "North Africa" OR "North African" OR "Northern Africa" OR "Northern African" OR "South African" OR "Southern Africa" OR "Southern African" OR "sub Saharan Africa" OR "sub Saharan African" OR "subSaharan Africa" OR "subSaharan African") NOT ("guinea pig" OR "guinea pigs" OR "aspergillus niger")

3. \#\#1 AND \#2

4. Date limits: 1 January 1986 to 30 October 2017, with no language restrictions 


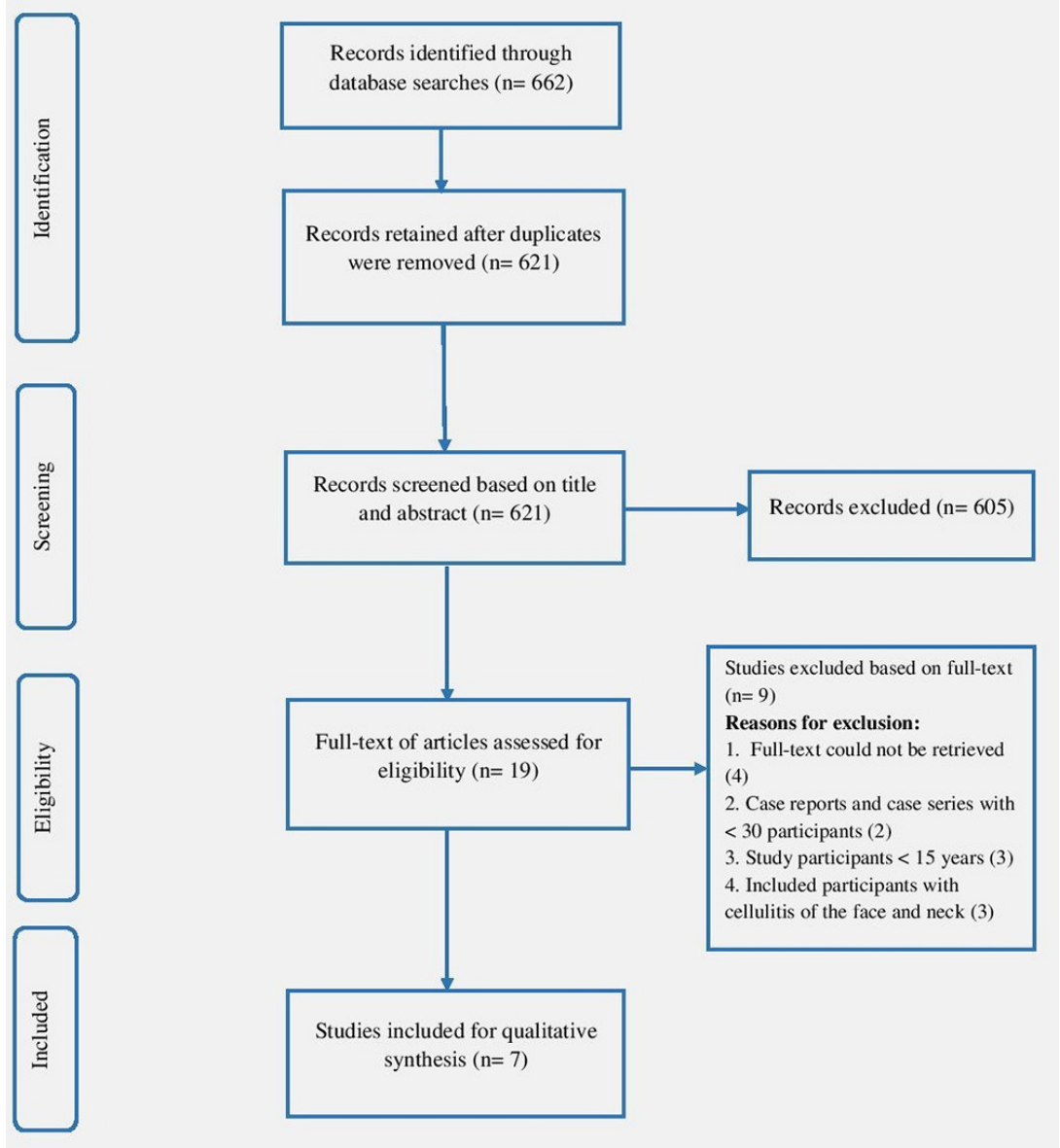

Figure 1 Flow diagram for study screening, selection and inclusion.

\section{Screening}

The screening process was conducted by two authors (VNA and CD) who independently screened the titles and abstracts of articles retrieved from the search, and recovered the full text of potentially eligible articles. Fulltext eligibility was then assessed independently by the aforementioned authors for final inclusion. Both authors resolved disagreements by discussion and consensus. Where a consensus was not reached, arbitration was done by a third author (F-LT). The process of screening, selection and inclusion of eligible articles and reasons for exclusion is displayed in figure 1.

\section{Data items, extraction and synthesis}

Relevant data from eligible full texts were extracted by two authors (CD and VNA) using prestructured data abstraction sheets. Three data abstraction sheets were predesigned to extract available data on the risk factors, complications and factors associated with complications of lower limb cellulitis in Africa. Disagreements were resolved as detailed above.

\section{General data items}

The following data items were extracted for included studies: Surname of the first author and year of publication; the country/countries in which the study was conducted in; the region (Northern, central, Southern,
Eastern or Western Africa); study area (urban, semiurban or rural); study design (cross-sectional, case control, case series or cohort); study setting (community-based or hospital-based); data collection (prospective or retrospective); random sampling (yes or no); male proportion; mean or median age in years and sample size.

\section{Risk factors of lower limb cellulitis}

In addition to the data items listed above, the adjusted OR $(\mathrm{aOR})$ and $95 \% \mathrm{CI}$ of significant risk factors of cellulitis were also extracted.

\section{Complications of lower limb cellulitis}

The different complications of lower limb cellulitis alongside their relative proportions were extracted in addition to the general items listed above in order to summarise data on the complications of lower limb cellulitis.

\section{Factors associated with complications of lower limb cellulitis}

To summarise data on the factors associated with complications of lower limb cellulitis, the different factors, their respective OR (unadjusted OR) and the individual 95\% CI were extracted in addition to the general items listed above. Where both the crude and aOR were reported, the aOR was preferably extracted every time.

Available data for multinational studies could not be disaggregated according to the individual countries 
in which the study was conducted and were therefore presented as a single study.

\section{Assessment of methodological quality}

The Newcastle-Ottawa tool $^{19}$ was used to assess the risk of bias for case-control ${ }^{2021}$ and cohort studies $^{22-24}$ (online supplementary table S1), while an adapted version of the risk of bias assessment tool for prevalence studies developed by Hoy $e t a l^{25}$ was used to assess the risk of bias for cross-sectional studies ${ }^{26}$ (online supplementary table S2).

\section{Data synthesis and analysis}

Due to the small number and evident heterogeneity among the included studies reporting on a wide variety of risk factors, complications and factors associated with complications of lower limb cellulitis, the findings herein were presented using tables and narrative summaries.

\section{Patient and public involvement}

Patients and/or the public were not directly involved in this study.

\section{RESULTS}

\section{Review process}

A summary of the selection process is presented in figure 1. A total of 662 records were retrieved from electronic database searches. After keeping out duplicates, the titles and abstracts of 621 articles were screened, from which 19 full-text articles were retained for review. Seven of these full-text articles met our inclusion criteria and were considered in the final review. ${ }^{20-24} 2627$

\section{Characteristics of included studies}

The characteristics of the studies included in our review are summarised in table 2. A total sample of 3031 participants were included, among which were 1973 cases of lower limb cellulitis. ${ }^{20-242627}$ The risk of bias was judged to be low, moderate and high in three, ${ }^{20-22}$ three ${ }^{24} 27$ and one study, ${ }^{26}$ respectively.

The included studies were published between the years 2006 and $2017^{20-24} 2627$; four of which were published in 2017. ${ }^{21242627}$ Sub-Saharan Africa was the most represented with four out of the seven included studies. ${ }^{21-24}$ All studies were conducted in a hospital setting with data collected prospectively in five studies ${ }^{20-24}$ and retrospectively in two studies. ${ }^{26} 27$ There was an overall male predominance ${ }^{20242627}$ with only three studies reporting a greater proportion of female participants. ${ }^{21-23}$ The age range and the median ages of the participants spanned from 15 to 88 years and 43.7 to 73 years, respectively. ${ }^{20-242627}$

\section{Risk factors of lower limb cellulitis}

One cohort study ${ }^{22}$ and two case-control studies ${ }^{20} 21$ reported on the risk factors of lower limb cellulitis, which were classified as local and general risk factors.

The local risk factors of lower limb cellulitis reported from the studies included: cutaneous barrier disruption
(aOR 13.6; 95\% CI 6.3 to 31), leg oedema (aOR 7.0; 95\% CI 1.3 to 38 ), history of skin disruption (aOR 12.4; $95 \%$ CI 3.9 to 39.1), presence of toe-web intertrigo (aOR 51.4, 95\% CI 11.7 to 225.6), lymphoedema (aOR 3.9; $95 \%$ CI 2.2 to 6.9 ), cosmetic depigmentation practices (aOR 4.3; 95\% CI 2.4 to 7.8 ) and traumatic wounds (aOR 37.9; $95 \%$ CI 24.9 to 57.7 ), table 3 . On the other hand, obesity (aOR 4.7, 2.8; 95\% CI 1.5 to $14.7,2.1$ to 3.8 , respectively) was the only reported general risk factor for lower limb cellulitis.

\section{Complications of lower limb cellulitis}

Five studies reported on the complications of lower limb cellulitis $^{21} 23242627$ of which three provided data on the factors associated with these complications. ${ }^{24} 2627$ The overall prevalence of complications (any complication) ranged from $8.9 \%$ to $47.4 \%$, table 4 . Complications of cellulitis were classified as local and general complications. The local complications of lower limb cellulitis were: leg abscesses, necrotising fasciitis, bullae, haemorrhagic lesions, necrosis, amputations, necrosectomy, skin abscesses and phlebitis, table 4 . Diabetic ketoacidosis was the only general complication of cellulitis, occurring in $1.9 \%$ of cases. ${ }^{26}$

\section{Factors associated with complications of lower limb cellulitis}

The following factors were associated with the complications of lower limb cellulitis: nicotine addiction (aOR 3.7; $95 \%$ CI 1.35 to 10.7 ), delay in the initiation of antibiotic treatment $\geq 10$ days (aOR $4.6 ; 95 \%$ CI 1.84 to 11.80 ), use of antibiotics (aOR 5.15; 95\% CI 1.28 to 20.72 ) or NSAIDs (OR 2.5; 95\% CI 1.41 to 4.45) before consultation, and an accelerated erythrocyte sedimentation rate (ESR) on admission (aOR 1.03; 95\% CI 1.00 to 1.06), table 4 .

\section{DISCUSSION}

This systematic review presents a summary of the risk factors, complications and factors associated with the complications of lower limb cellulitis in Africa, based on data from seven studies involving 3031 participants. Obesity was the only identified general risk factor for lower limb cellulitis in Africa. Meanwhile local risk factors included: cutaneous barrier disruption, leg oedema, presence of toe-web intertrigo, lymphoedema, cosmetic depigmentation practices and traumatic wounds. Complications occurred in $8.9 \%-47.4 \%$ of cases of lower limb cellulitis, which included: leg abscess, necrotising fasciitis, bullae, haemorrhagic lesions, necrosis, skin abscess, phlebitis and diabetic ketoacidosis. On the other hand, nicotine addiction, delay in antibiotic treatment for 10 days or more, use of NSAIDs or antibiotics before consultation and accelerated ESR on admission were associated with these complications. ${ }^{20-24} 2627$

The median age of 43.7-73 years indicates that lower limb cellulitis predominantly affects individuals in Africa between their mid-forties and mid-seventies, ${ }^{29}$ and it poses a greater burden to this elderly population where 


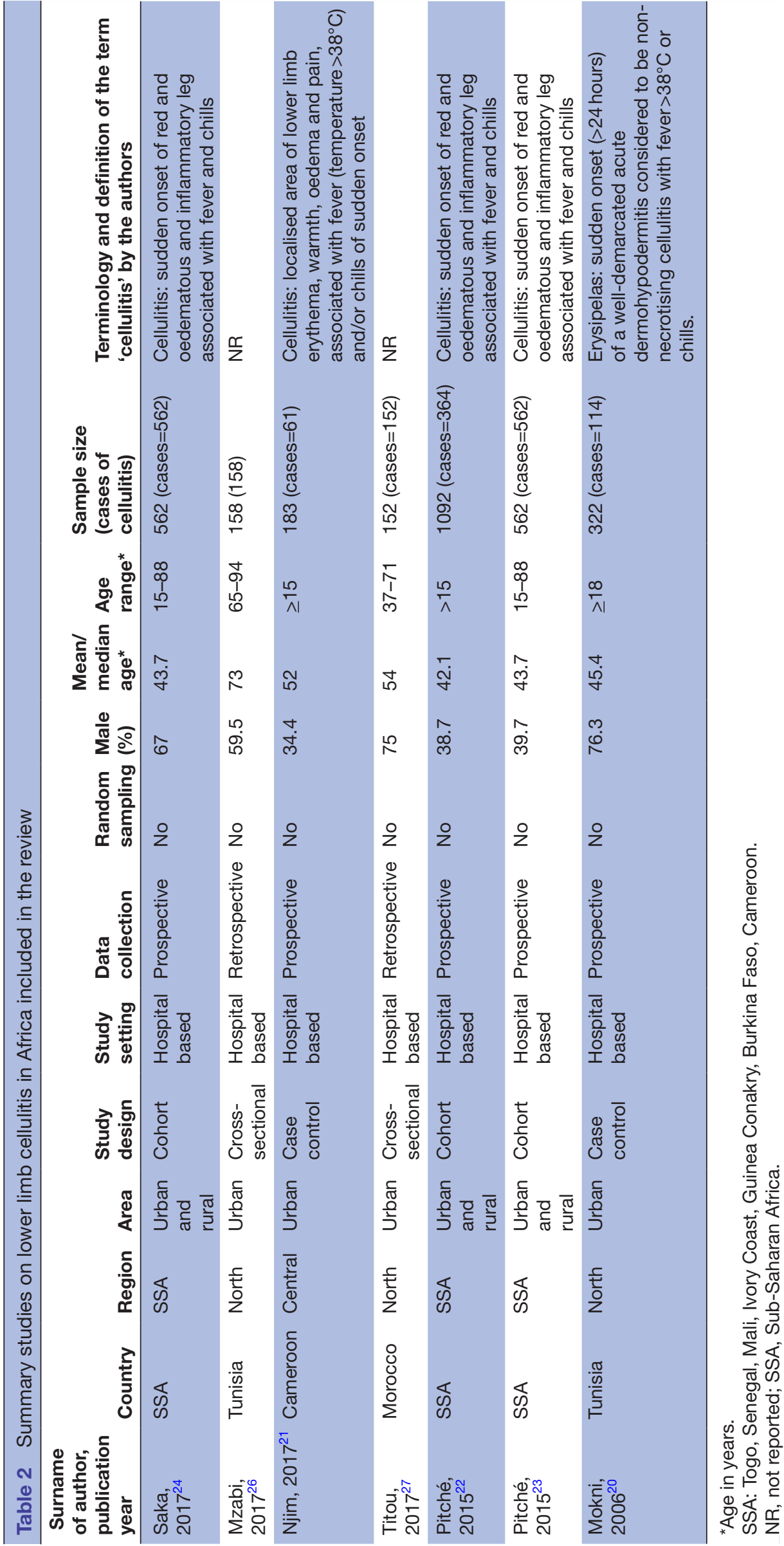

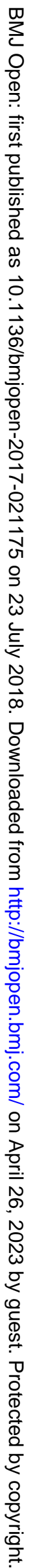




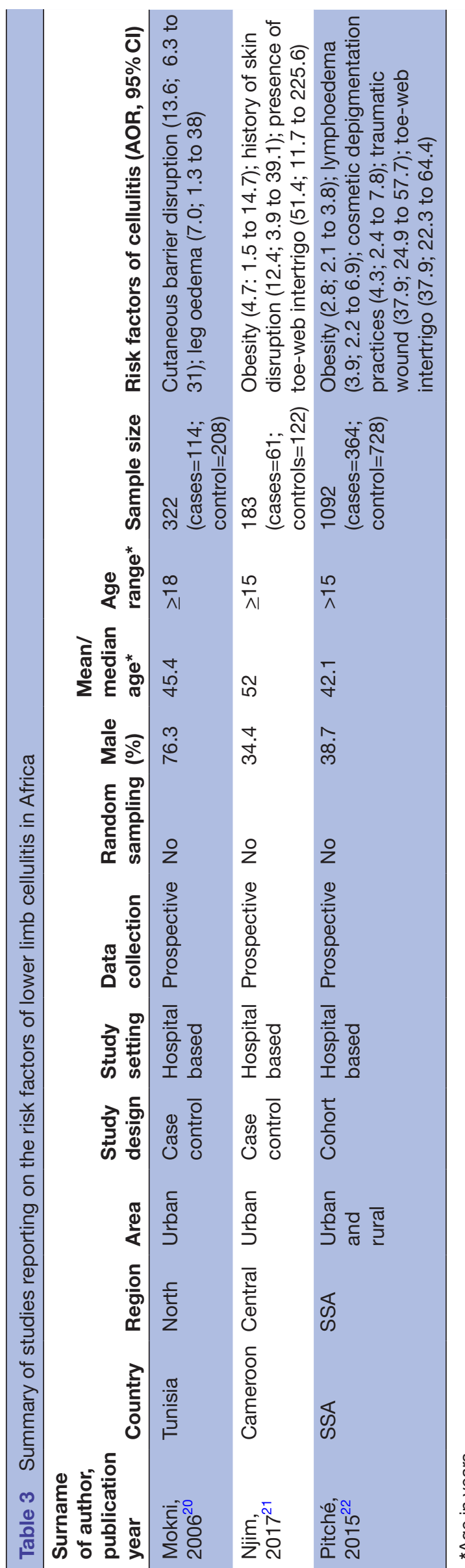

comorbid conditions, such as hypertension, diabetes mellitus, heart failure and venous insufficiency, are not a rarity. ${ }^{30}$

A break in the physical barrier of the skin seems to be a major determinant for developing cellulitis in Africa. A bridge in the skin barrier serves as a potential portal of entry for cellulitis causing pathogens. ${ }^{5629}$ Consequently, factors which disrupt the physical skin barrier, such as the presence of toe web-intertrigo, traumatic and neglected wounds, have been consistent as risk factors of lower limb cellulitis in Africa and elsewhere. ${ }^{13} 1431$ Voluntary skin depigmentation, although poorly studied, seems to be becoming an emerging risk factor of lower limb cellulitis in Africa; as the practice is rapidly spreading among African women, as well as some men. ${ }^{32-34}$ Most of these products contain potent corticosteroids which cause skin atrophy, dyschromia, hypertrichosis and striae in the long run. The resulting fragile skin is more susceptible to penetration and colonisation by cellulitis causing pathogens. ${ }^{32} 33$ This practice is increasingly being associated with lower limb cellulitis in African women and is a major cause of morbidity as systemic absorption of these potent corticosteroids could result in hypertension, diabetes and adrenal insufficiency. ${ }^{35}$

The only general risk factor for lower limb cellulitis in our review was obesity. This was similar to findings from Quirke et $a l^{16}$ Obesity-related immune system dysfunction with a decreased cell-mediated immune response could predispose obese individuals to developing cellulitis. ${ }^{36}$ Obesity is also associated with other comorbid conditions, such as diabetes mellitus and hypertension, which may otherwise influence the risk for lower limb cellulitis. ${ }^{37}$

With the increasing prevalence of obesity in Africa, owing to westernisation and urbanisation, and the increasing number of persons practising voluntary skin depigmentation, the burden of cellulitis in Africa, is likely to increase in subsequent years and therefore warrants surveillance and control. ${ }^{24} 333438$ Sadly, cellulitis is still given very little consideration in Africa, as is evident by the limited number of studies on the subject in this part of the world. Majority of the identified risk factors of cellulitis are potentially modifiable. ${ }^{14} 1621$ In fact, Njim et al identified the presence of toe-web intertrigo and a history of disruption in skin barriers to have a high population-attributable risk among independent modifiable risk factors of lower limb cellulitis. ${ }^{21}$ Identifying and improving the management of these preventable factors could go a long way in decreasing the morbidity and health costs incurred by this condition. ${ }^{13}$ Routine screening and appropriate management of toe-web intertrigo, obesity and other local risk factors could contribute significantly in reducing the burden of lower limb cellulitis in Africa. ${ }^{13}{ }^{14}$ Encouraging adequate foot wear, foot hygiene and self-foot examination to prevent toe-web intertrigo 5829 39; promoting healthy feeding habits and exercise to reduce the incidence of cellulitis; and inspiriting recommended healthcare-seeking habits in case of 


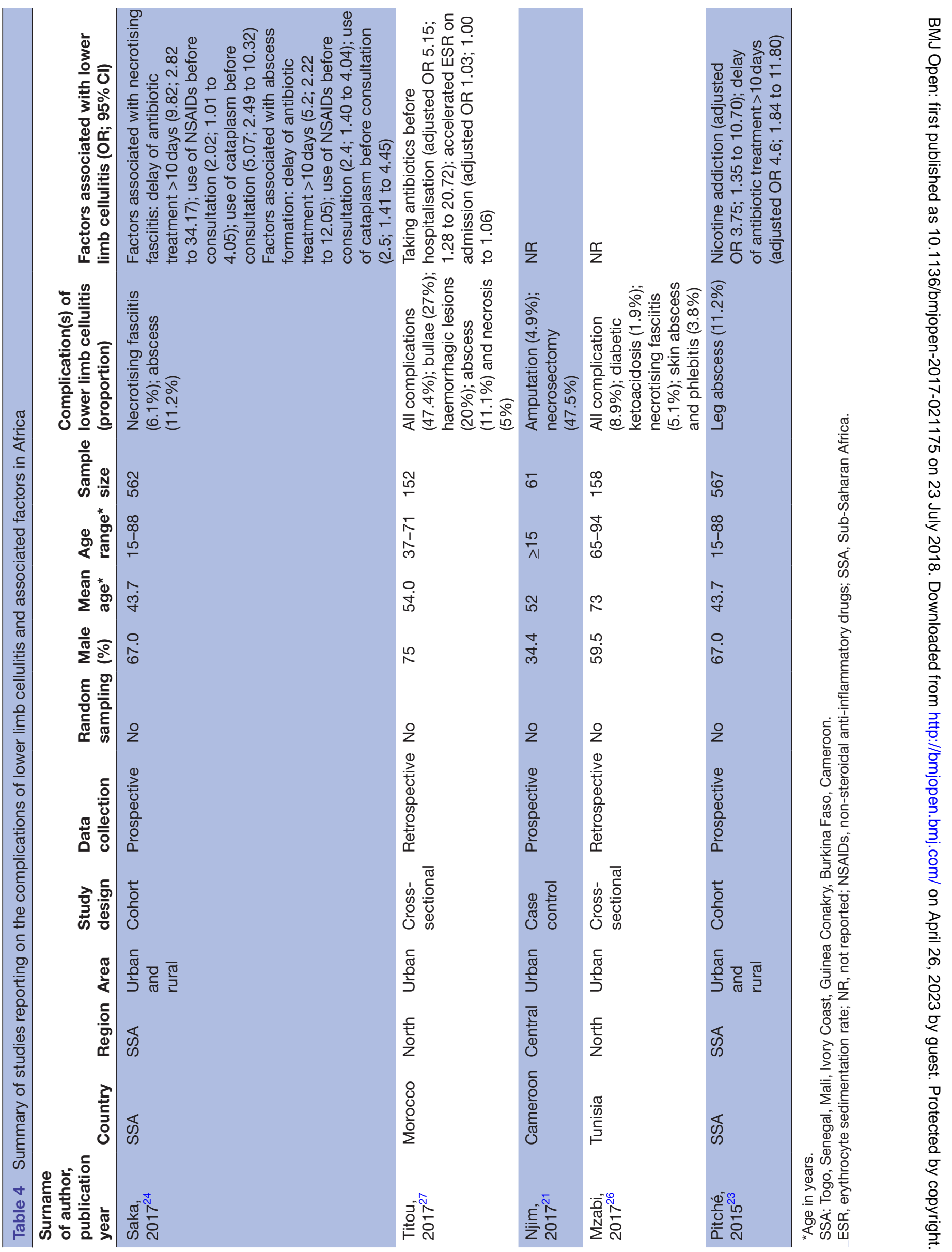


a wound, seem appropriate to reduce the occurrence of this disease at community level.

Leg abscesses and necrotising fasciitis were the most frequently reported complications of cellulitis. ${ }^{23242627} \mathrm{~A}$ delay in antibiotic therapy for 10 days or more, nicotine addiction and use of NSAIDS, antibiotics or cataplasm prior to consultation were associated with the occurrence of local complications of lower limb cellulitis, table 4 . These findings are similar to other studies done elsewhere. ${ }^{82829}$ With the poor healthcare-seeking behaviour in Africa, the patient with a neglected wound or early signs of cellulitis will make a first stop at a local chemist shop or traditional herbalist where a cataplasm will be placed and/or they will be administered some antibiotics and anti-inflammatories, typically an NSAID, for their symptoms. These pose a barrier to early and adequate diagnosis and treatment of lower limb cellulitis in Africa, predisposing them to debilitating and life-threatening complications of the disease. ${ }^{29} 3341$ The occurrence of complications of lower limb cellulitis prolongs the evolution of the disease and usually results in longer hospital stays, increased morbidity and increased healthcare costs on both the individual and the public health sector. ${ }^{9} 1328$ These complications often require surgical interventions such as incisions and drainage, necrosectomies and amputations in extreme cases. ${ }^{80}$ Therefore, it is vital for clinicians to have good knowledge on the complications of lower limb cellulitis and the factors associated with these complications, so as to be better equiped to provide optimal and targeted care to at-risk patients, in a bid to curb the burden associated with these complications.

The authors will like to highlight some limitations which should be taken into consideration during the global appraisal of the findings herein. All of the studies included in this review were hospital-based, limiting inference of our findings to the general population. This limited the cases of cellulitis to those deemed severe enough by patients to require health intervention. In addition, the very small number of included studies and the exclusion of four potentially eligible studies as their full texts could not be retrieved is likely to skew the findings presented in this review. Over $30 \%$ of cases of cellulitis have an alternative diagnosis. ${ }^{42}$ This is a potential limitation to our study, especially from retrospective studies. However, this is the first systematic review summarising the risk factors, complications and the factors associated with the complications of lower limb cellulitis in Africa. Also, the inclusion of recent studies provides a contemporaneous picture of the risk factors and complications of cellulitis in Africa.

\section{CONCLUSION}

This review highlights the important role of local risk factors in the pathogenesis of lower limb cellulitis in Africa. Voluntary skin depigmentation is gaining popularity among African women and its association with lower limb cellulitis should alert public health authorities and the general population to the health risks associated with this practice. Leg abscesses and necrotising fasciitis seem to be the major complications of lower limb cellulitis in Africa, with a delay in the initiation of antibiotherapy of 10 days or more, use of NSAIDs, antibiotics and cataplasm prior to consultation all being associated with the occurrence of these complications. Identification and management of preventable risk factors of lower limb cellulitis could go a long way to curb morbidity and health costs incurred by lower limb cellulitis in the region.

\section{RESEARCH PERSPECTIVES}

Cellulitis still remains a virgin area of research in Africa. Population-based studies are needed to evaluate the prevalence of cellulitis among African countries and better estimate the disease burden and plan control measures. Prospective case-control and cohort studies with larger sample sizes are warranted to adequately describe the risk factors and complications associated with cellulitis in Africa. The health effects of voluntary skin depigmentation need further assessment to better evaluate the health risks of this practice in the region. Also, more precise estimates of the population-attributable risk of the risk factors of cellulitis are needed to guide policy-makers in implementing preventive strategies both at health facility and community level. In addition, bacteriological studies on the causative agents of cellulitis in Africa are scarce. Finally, studies evaluating the benefits of systematic screening and treatment of toe-web intertrigo as a preventive measure of cellulitis are justified.

Contributors VNA conceived the study and searched data. VNA and CD screened and selected eligible full texts. CD and VNA extracted the data. CD, F-LT, CMM and VNA interpreted the data. CMM, FL-T and VNA drafted the initial manuscript. CD, F-LT, CMM and VNA revised the manuscript for intellectual content. All authors approved the final version of the manuscript. VNA is the guarantor of the review.

Funding The authors have not declared a specific grant for this research from any funding agency in the public, commercial or not-for-profit sectors.

Competing interests None declared.

Patient consent Not required.

Provenance and peer review Not commissioned; externally peer reviewed.

Data sharing statement Available data can be obtained by contacting the corresponding author.

Open access This is an open access article distributed in accordance with the Creative Commons Attribution Non Commercial (CC BY-NC 4.0) license, which permits others to distribute, remix, adapt, build upon this work non-commercially, and license their derivative works on different terms, provided the original work is properly cited, appropriate credit is given, any changes made indicated, and the use is non-commercial. See: http://creativecommons.org/licenses/by-nc/4.0/.

\section{REFERENCES}

1. Bisno AL, Stevens DL. Streptococcal infections of skin and soft tissues. N Engl J Med 1996;334:240-5.

2. Gabillot-Carré M, Roujeau JC. Acute bacterial skin infections and cellulitis. Curr Opin Infect Dis 2007;20:118-23.

3. Bonnetblanc JM, Bédane C. Erysipelas: recognition and management. Am J Clin Dermatol 2003;4:157-63.

4. Phoenix G, Das S, Joshi M. Diagnosis and management of cellulitis. BMJ 2012;345:e4955. 
5. Crickx B, Chevron F, Sigal-Nahum M, et al. [Erysipelas: epidemiological, clinical and therapeutic data (111 cases)]. Ann Dermatol Venereol 1991;118:11-16.

6. Bernard P, Chosidow O, Vaillant L; French Erysipelas Study Group. Oral pristinamycin versus standard penicillin regimen to treat erysipelas in adults: randomised, non-inferiority, open trial. BMJ 2002;325:864

7. Chartier C, Grosshans E. Erysipelas. Int J Dermatol 1990;29:459-67.

8. Jorup-Rönström C. Epidemiological, bacteriological and complicating features of erysipelas. Scand $J$ Infect Dis 1986;18:519-24.

9. Krasagakis K, Samonis G, Valachis A, et al. Local complications of erysipelas: a study of associated risk factors. Clin Exp Dermatol 2011;36:351-4.

10. Africa CiS;Pages. http:// global-disease burden.healthgrove.com/l/ 82932/Cellulitis-in-South-Africa

11. Kassebaum NJ, Arora M, Barber RM, et al. Global, regional, and national disability-adjusted life-years (DALYs) for 315 diseases and injuries and healthy life expectancy (HALE), 1990-2015: a systematic analysis for the Global Burden of Disease Study 2015. The Lancet 2016;388:1603-58.

12. Transforming our world: the 2030 Agenda for Sustainable Development. Sustainable Development Knowledge Platform. https://sustainabledevelopment.un.org/post2015/ transformingourworld.

13. Björnsdóttir S, Gottfredsson M, Thórisdóttir AS, et al. Risk factors for acute cellulitis of the lower limb: a prospective case-control study. Clin Infect Dis 2005;41:1416-22.

14. Dupuy A, Benchikhi H, Roujeau JC, et al. Risk factors for erysipelas of the leg (cellulitis): case-control study. BMJ 1999;318:1591-4.

15. Khawcharoenporn T, Tice AD, Grandinetti A, et al. Risk factors for community-associated methicillin-resistant Staphylococcus aureus cellulitis--and the value of recognition. Hawaii Med J 2010;69:232.

16. Quirke M, Ayoub F, McCabe A, et al. Risk factors for nonpurulent leg cellulitis: a systematic review and meta-analysis. Br J Dermatol 2017; 177:382-94.

17. Moher D, Liberati A, Tetzlaff J, et al. Preferred reporting items for systematic reviews and meta-analyses: the PRISMA statement. PLOS Med 2009;6:e1000097.

18. Pienaar E, Grobler L, Busgeeth $\mathrm{K}$, et al. Developing a geographic search filter to identify randomised controlled trials in Africa: finding the optimal balance between sensitivity and precision. Health Info Libr J 2011;28:210-5.

19. Wells G, Shea B, O'connell D, et al. The Newcastle-Ottawa Scale (NOS) for assessing the quality of nonrandomized studies in metaanalysis. 2011. http://www. ohri. ca/programs/clinical_epidemiology/ oxford.asp.2016

20. Mokni M, Dupuy A, Denguezli M, et al. Risk factors for erysipelas of the leg in Tunisia: a multicenter case-control study. Dermatology 2006;212:108-12.

21. Njim T, Aminde LN, Agbor VN, et al. Risk factors of lower limb cellulitis in a level-two healthcare facility in Cameroon: a case-control study. BMC Infect Dis 2017;17:418.

22. Pitché P, Diatta B, Faye O, et al. [Risk factors associated with leg erysipelas (cellulitis) in sub-Saharan Africa: A multicentre casecontrol study]. Ann Dermatol Venereol 2015;142:633-8.
23. Pitché PV, Saka B, Diatta AB, et al. Risk factors associated with abscess formation among patient with leg erysipelas (cellulitis) in sub-Saharan Africa: a multicenter study. BMC Dermatol 2015;15:18.

24. Saka B, Diatta AB, Faye O, et al. Erysipelas of the leg (cellulitis) in sub-Saharan Africa: a multicentric study of 562 cases. Our Dermatol Online 2017;8

25. Hoy D, Brooks P, Woolf A, et al. Assessing risk of bias in prevalence studies: modification of an existing tool and evidence of interrater agreement. J Clin Epidemiol 2012;65:934-9.

26. Mzabi A, Marrakchi W, Alaya Z, et al. Cellulitis in aged persons: a neglected infection in the literature. Pan Afr Med J 2017;27:160.

27. Titou H, Ebongo C, Bouati E, et al. Risk factors associated with local complications of erysipelas: a retrospective study of 152 cases. Pan Afr Med J 2017;26:66.

28. Picard D, Klein A, Grigioni S, et al. Risk factors for abscess formation in patients with superficial cellulitis (erysipelas) of the leg. $\mathrm{Br} J$ Dermatol 2013;168:859-63.

29. Pitche P, Tchangaï-Walla K. [Erysipelas of the leg in hospital environment in Lome (Togo)]. Bull Soc Pathol Exot 1997;90:189-91.

30. Agbor VN, Essouma M, Ntusi NAB, et al. Heart failure in sub-Saharan Africa: A contemporaneous systematic review and meta-analysis. Int J Cardiol 2018;257:207-15.

31. Roujeau JC, Sigurgeirsson B, Korting HC, et al. Chronic dermatomycoses of the foot as risk factors for acute bacterial cellulitis of the leg: a case-control study. Dermatology 2004;209:301-7.

32. Cisse M, Keïta M, Toure A, et al. Dermohypodermites bactériennes: étude monocentrique rétrospective de 244 cas observés en Guinée. Annales de Dermatologie et de Vénéréologie 2007;134:748-51.

33. Gathse A, Ntsiba $H$. Etude rétrospective de 53 érysipèles hospitalisés au CHU de Brazzaville, Congo. Bulletin de la Société de pathologie exotique 2006;99:3-4.

34. Thierno M, Diop N, Niang S, et al. Dermohypodermite bactérienne et dépigmentation artificielle: A propos de 60 cas observés au Sénégal. Les Nouvelles dermatologiques 2001;20:630-2.

35. Couteau C, Coiffard L. Overview of Skin Whitening Agents: Drugs and Cosmetic Products. Cosmetics 2016;3:27.

36. Falagas ME, Athanasoulia AP, Peppas G, et al. Effect of body mass index on the outcome of infections: a systematic review. Obes Rev 2009;10:280-9.

37. Huttunen R, Syrjänen J. Obesity and the risk and outcome of infection. Int J Obes 2013;37:333.

38. Adeboye B, Bermano G, Rolland C. Obesity and its health impact in Africa: a systematic review. Cardiovasc J Afr 2012;23:512-21.

39. George AO. Skin Diseases in Tropical Africa. International Journal of Dermatology 1988;27:187-9.

40. Crickx B. [Erysipelas: evolution under treatment, complications]. Ann Dermatol Venereol 2001;128(3 Pt 2):358-62.

41. Saka B, Kombaté K, Mouhari-Toure A, et al. [Bacterial dermohypodermitis and necrotizing fascitis: 104-case series from Togo]. Med Trop 2011;71:162-4.

42. Weng QY, Raff AB, Cohen JM, et al. Costs and Consequences Associated With Misdiagnosed Lower Extremity Cellulitis. JAMA Dermatol 2016. 